\title{
Genetic analysis of grain yield and yield-attributing traits in navy bean (Phaseolus vulgaris L.) under drought and optimal environments
}

\author{
Bruce Mutari ( $\nabla$ brucemutari@gmail.com ) \\ University of KwaZulu-Natal - Pietermaritzburg Campus https://orcid.org/0000-0002-4159-287X \\ Julia Sibiya \\ University of KwaZulu-Natal - Pietermaritzburg Campus \\ Edmore Gasura \\ University of Zimbabwe \\ Prince Muchapondwa Matova \\ Department of Research and Specialist Services \\ Kennedy Simango \\ Marondera University of Agricultural Sciences and Technology \\ Aleck Kondwakwenda \\ University of KwaZulu-Natal - Pietermaritzburg Campus
}

\section{Research Article}

Keywords: Combining ability, drought tolerance, diallel analysis, heritability, yield components

Posted Date: November 30th, 2021

DOI: https://doi.org/10.21203/rs.3.rs-1056631/v1

License: @ (i) This work is licensed under a Creative Commons Attribution 4.0 International License. Read Full License 


\section{Abstract}

Knowledge of the genetic basis of navy bean (Phaseolus vulgaris L.) performance under drought stress (DS) is important for planning appropriate breeding and selection strategies in DS environments. Twenty-eight $F_{2}$ progenies generated from an $8 \times 8$ half-diallel mating design were evaluated to determine combining ability effects and mode of gene action of grain yield (GYD) and yield attributing traits in navy bean under DS and nonstressed (NS) conditions. The experiments were conducted in two locations in a $6 \times 6$ square lattice design with two replications during the 2020 dry season. There were significant $(p<0.001 ; p<0.05)$ positive correlations for number of pods per plant (NPPP), number of seeds per plant (NSPP) and 100-seed weight (SW) with GYD under both DS and NS. General and specific combining ability (GCA; SCA) effects were significant $(p<0.05)$ under both DS and NS for most traits indicating the importance of both additive and non-additive gene effects in the expression of the traits. Parents with best combining ability for most of the studied traits were G1, G7, G6 and G8 under NS, and G3, G4, G7 and G8 under DS. The most promising progenies with high values for GYD and its component traits under DS were G2 X G3, G2 X G8, G4 X G5, G4 X G8, and G6 X G8. Good general and specific combiners with high significant positive effects under DS should be used further in breeding for moisture stress tolerance.

\section{Introduction}

Navy bean (Phaseolus vulgaris L.) is an important food legume crop in Zimbabwe, grown mostly for human consumption and income generation. It is mainly consumed as canned beans making it an essential raw material for the bean canning industry in Zimbabwe, which requires around 4000 MT of grain annually (Mukweza, personal communication, May 2018). However, local supplies can meet only $40 \%$ of the national consumption requirements (Tsiko 2020). Despite its importance in human diets and the bean canning industry, average grain yields realised by smallholder farmers in Zimbabwe have remained relatively low (less than $700 \mathrm{~kg} \mathrm{ha}^{-1}$ ) (AGRITEX 2016) against a yield potential of $3000 \mathrm{~kg} \mathrm{ha}^{-1}$. The large discrepancy in yields has been attributed to the different biotic and abiotic stresses, including drought stress (Rainey and Griffiths 2003; Rainy and Griffiths 2005; Porch et al. 2007; Beebe et al. 2013; Katungi et al. 2017; Mutari et al. 2021).

Some of the navy bean farmers in Zimbabwe produce the crop in drought-prone areas under rain-fed conditions (January - April) with no capacity for supplemental irrigation during periods of drought stress (Mutari et al. 2021). Moreover, the rainfall pattern fluctuates from season to season due to climate change, consequently exposing the crop to moisture stress (intermittent or terminal) at some stage during growth. This severely affects productivity and grain quality of navy bean. The recent participatory rural appraisal study conducted by Mutari et al. (2021) in the main navy bean growing regions of Zimbabwe revealed that the crop experiences moisture stress during the reproductive stage of development. Singh (2001) and Beebe et al. (2010) reported that about $60 \%$ of cultivated beans worldwide are grown under the risk of either terminal or intermittent drought. The impact of drought stress to navy bean productivity in Zimbabwe is further worsened by lack of drought tolerant cultivars (Mutari et al. 2021). Therefore, the development, commercial release, and subsequent cultivation of drought tolerant cultivars would be an effective, sustainable, and appropriate strategy for ensuring increased navy bean productivity and stability to ensure self-sufficiency and food security in Zimbabwe.

To develop improved genotypes that are adapted to drought stress with significant genetic gain, knowledge of genetic variability of drought stress tolerance and its genetic basis is important. Several studies have reported that genetic variability for drought stress tolerance exists in dry beans (Assefa et al. 2013; Makunde 2013; Darkwa et al. 2016), implying that genetic improvement of this trait would be possible. Considering that grain yield is a quantitative polygenic trait, selection of grain yield along with its attributing traits could be more reliable and efficient than selection on the basis of grain yield alone (Romanus et al. 2008). Thus, selection efficiency in dry bean improvement programs can be improved by generating information on the association between grain yield and yield attributing traits.

Information on the combining ability and genetic control of economic quantitative traits such as grain yield and its associated traits under drought stress environments is cardinal when improving crops for drought tolerance. Combining ability is the breeding value of parental genotypes to produce superior hybrids based on the performance of their offspring (Romanus et al. 2008). General combining ability refers to the average performance of a parental line in a series of cross-combinations (Sprague and Tatum 1942; Griffings 1956). On the other hand, specific combining ability is the average performance of certain cross-combinations relatively better or poorer than would be expected on the basis of the average performance (GCA) of the other parental lines involved (Su et al. 2017). Combining ability analysis assists bean breeders in the identification of the best hybrid combinations with high SCA and parental lines with high GCA, increasing the chances of selecting superior transgressive segregants in the subsequent segregating populations. It also provides information on the type of gene action governing the expression of different quantitative agronomic traits of interest.

Evidence for the expression of grain yield and its components in beans under drought stressed (DS) and non-drought stressed (NS) environments is contradictory. Phiri (2015) reported the predominance of additive gene action over the non-additive gene action in controlling the number of pods per plant, number of days to $50 \%$ flowering, number of seeds per pod, hundred seed weight except for grain yield under DS environments. Asadi et al. (2010) and Amongi et al. (2015) reported that drought tolerance is governed by both additive and non-additive genes with predominance of additive gene effects for grain yield, pod weight, number of seeds per pod and number of pods per plant. On the contrary, Makunde et al. (2007) reported the predominance of dominance effects for grain yield, number of days to $95 \%$ flowering and physiological maturity under both DS and NS conditions. These differences necessitate the need to conduct genetic analysis studies for germplasm to be used for specific breeding programmes to elucidate the nature of gene action governing the inheritance of grain yield and yield related traits under moisture stressed conditions. The 
objectives of this study were to (i) estimate the combining ability effects and gene action for grain yield and its attributing traits among F2 progenies under DS and NS conditions and (ii) determine association between grain yield and yield attributing traits under DS and NS conditions, in order to assess the feasibility of indirect selection for grain yield.

\section{Materials And Methods}

Experimental materials and study locations

Eight parental lines comprising of three drought tolerant genotypes (ZABRA16575-73F22, G37 and DAB550), three drought susceptible genotypes (CZ113-13, G6 and G97) and two other genotypes (CIM-NAV02-17-3 and Navy Line60) with desirable agronomic traits that include high grain yield, early maturity, resistance to diseases and pod shattering were used (Table 1).

Table 1

Description of selected characteristics of the eight parents used in the study

\begin{tabular}{|lll|}
\hline Parent & Abbreviation & Characteristic \\
\hline CZ113-13 & G1 & Tolerant to pod shattering but susceptible to drought stress \\
\hline CIM-NAV02-17-3 & G2 & Tolerant to diseases of economic importance but susceptible to moisture stress \\
\hline ZABRA16575-73F22 & G3 & Tolerant to moisture stress and tolerant to diseases of economic importance \\
\hline G37 & G4 & Early maturing and high yielding \\
\hline G6 & G5 & High yielding but susceptible to moisture stress \\
\hline Navy Line60 & G6 & Tolerant to moisture stress and high yielding \\
\hline G97 & G7 & High grain yield potential but susceptible to moisture stress \\
\hline DAB550 & G8 & Tolerant to moisture stress and early maturing \\
\hline
\end{tabular}

The moisture stress tolerant and susceptible genotypes were selected based on previous evaluations under contrasting water regimes by the national bean breeding programme in Zimbabwe (unpublished work). The lines were obtained from the International Centre of Tropical Agriculture (CIAT) in Malawi. Hybridizations were conducted at Crop Breeding Institute (CBI) between July 2019 and November 2019). Field evaluation experiments were conducted at Chiredzi Research Station (CRS) and Chisumbanje Experiment Station (CES) in Zimbabwe during the dry season between May 2020 to August 2020. Both CRS (latitude -20.23369, longitude 30.72197 and altitude 494 meters above sea level (masl)) and CES (latitude -20.79966, longitude 32.23361 and altitude 413 masl) are located in the lowveld region, which is generally dry and warm during the dry season. Chisumbanje Experiment Station is characterized by basalt clay soils, whereas CRS has sandy clay loam soils. The weather conditions that were recorded during the study period at CRS and CES are summarized in Online Resource 1. Notably, no rainfall was recorded during the duration of the field experiment since the trials were conducted during the dry season.

Generation of filial generations, trial establishment, and management

At flowering, the parents were cross pollinated inside a glasshouse in all possible cross-combinations using an $8 \star 8$ half diallel mating design, Method II, following the artificial hybridization procedure of Makunde (2007). The twenty-eight $F_{1}$ crosses, along with eight parents, were left to selfpollinate in a glasshouse from January 2020 to April 2020 to produce sufficient seed for genetic analysis at the $F_{2}$ generation in replicated field trials. A total of eight parents and twenty-eight $F_{2}$ progenies were evaluated at CRS and CES under DS and NS (control) environments in a $6 * 6$ square lattice design with two replications, in two locations. Each genotype was planted in four row plots, $3 \mathrm{~m}$ long and $0.45 \mathrm{~m}$ wide. Intra-row spacing was $0.20 \mathrm{~m}$. In the DS treatments, the genotypes were irrigated with overhead sprinkler irrigation system up to when $80 \%$ of the plants had flowered and thereafter moisture stress was imposed until physiological maturity to induce terminal drought stress (Assefa et al. 2013; Beebe et al. 2013). This was done by withholding irrigation to $30 \%$ of the field capacity before re-watering based on tensiometer reading (Darkwa et al. 2016). Under the NS treatments, soil moisture was maintained at field capacity from planting until physiological maturity.

Data collection

The data for number of days from planting to $50 \%$ flowering (DFW), number of days of seed fill (DSF), and number of days from planting to physiological maturity (DPM) were recorded from two middle rows of each plot excluding the boarder plants on the ends of each row. At physiological maturity, plant height ( $\mathrm{PH} ; \mathrm{cm}$ ), number of pods per plant (NPPP), number of seeds per pod (NSP), number of seeds per plant (NSPP), and 100-seed weight (SW; g) were recorded as average of the ten randomly selected plants. Additionally, grain yield (GYD; g) was recorded at $14 \%$ moisture content based on ten plants per plot.

Statistical analyses

Page $3 / 17$ 
The collected data were subjected to analysis of variance (ANOVA) per environment and also across environment (combined ANOVA) using the Breeding Management Systems (BMS) statistical analysis software version 18 (The Integrated Breeding Platform's BMS 2021). Bartlett's test of homogeneity of error variance across the two locations was conducted for all traits (Bartlett 1947). The means were separated using the Least Significant Difference (LSD). In the combined ANOVA a mixed linear model was followed, genotypes were considered as fixed effects and the environment, blocks and replications were considered as random effects. Pearson's correlation analysis was performed using Genstat 18 th edition (Payne et al. 2018) to determine the degree of trait association within separate moisture treatments. The GCA and SCA effects were determined separately per environment according to Griffing's (1956) Method II, Model I using the Analysis of Genetic Designs in R software, version 3.0 (Rodriguez et al. 2015). The fixed model for combining ability analysis was as follows:

$Y_{i j k}=\mu+g_{i}+g_{j}+S_{i j}+\mathrm{rk}+e_{i j k}$

Where $Y_{i j k}$ is the mean phenotypic value of a character measured on cross i $\mathrm{j}$ in $k$ th replication, $\mu$ is the general/population mean effect, $g_{i}$ and $g_{j}$ are the GCA effects of $i^{\text {th }}$ and $j^{\text {th }}$ parental lines, respectively, $S i j$ is the SCA effect of $i x j$ crosses, rk is the replication effect and $\mathrm{e}_{\mathrm{ijk}}$ is the environmental effect associated with the $i j k^{\text {th }}$ individual observation (Griffing 1956; Dabholkar 1992). The significance of variance due to GCA and SCA effects was tested using ' $\mathrm{t}$ ' test. To make inferences on the type of gene action involved in the expression of GYD and yield components, the relative importance of GCA and SCA was determined using Baker's ratio (Baker 1978) as follows:

$$
\text { Baker'sratio }=\frac{2 \mathrm{MS}_{\mathrm{GCA}}}{2 \mathrm{MS}_{\mathrm{GCA}}+\mathrm{MS}_{\mathrm{SCA}}}
$$

where: $\mathrm{MS}_{\mathrm{GCA}}$ is mean square for GCA, and $\mathrm{MS}_{\mathrm{SCA}}$ is mean square for SCA. The GCA: SCA ratio was estimated by dividing GCA with SCA using the sum of squares of the respective trait. To identify parental lines and $F_{2}$ progenies that combine high GYD with drought tolerance, percentage grain yield reduction (\% GYR) or drought tolerant index was calculated as the percentage of GYR due to moisture stress on the GYD obtained under NS environments as:

$$
\% G Y R=\left(\frac{\text { meangrainyieldofgenotypeunderNS }- \text { meangrainyieldofthesamegenotypeunderDS }}{\text { meangrainyieldofthesamegenotypeunderNS }}\right) \mathrm{X} 100
$$

Genotypes with good stability across both moisture stress and non-stress environments will have lower values of the \% GYR, while high values will indicate poor stability.

\section{Results}

Variation under moisture stressed and non-moisture stressed environments

The mean square values and significant tests for the nine traits of $28 \mathrm{~F}_{2}$ progenies and 8 parents evaluated across two water regimes and two locations are presented in Table 2. The mean square estimates for genotypes (G) were significant $(p<0.05)$ for DFW, DMP, NPPP, NSP, GYD and SW under both DS and NS environments. On the other hand, the mean square for genotypes was only significant $(p<0.05)$ for DSF and NSPP under DS conditions. Mean squares for location (L) were significant $(p<0.05)$ for NPPP, NSP and GYD under both DS and NS. Combined ANOVA showed high significant effects $(p<0.001)$ of the genotypes and locations on DPM, NPPP, and GYD. The effects of environmental conditions (water regime - WR; DS vs. NS) were significant $(p<0.05)$ for all the studied traits except for NSPP. Non-significant effects of the genotype $\mathrm{x}$ location interaction $(G \times L)$ were observed for all the traits except for NSP. However, the L X WR interaction significantly $(p<0.001)$ affected PH, DFW, DSF, NSP, and NSPP, while the $\mathrm{G} \times$ WR interaction effects were only significant $(p<0.05)$ for DSF, GYD, and SW.

Mean performance of genotypes under moisture stressed and non-moisture stressed environments

The means of parental lines and $F_{2}$ progenies with respect to GYD and its components are presented in Table 3 and Online Resource 2 . Generally, the average performance values of all the parents and $F_{2}$ progenies for all the traits under DS were lower than the mean performance of all the parents and $F_{2}$ progenies under NS conditions due to moisture stress. For instance, DPM decreased from 98.78 to 92.33 in the NS and DS experiments, respectively. A decrease in NPPP from 24.45 to 18.80 in the NS and DS experiments, respectively was observed. Grain yield also decreased from $2125 \mathrm{~g}$ under NS conditions to $1116 \mathrm{~g}$ under DS conditions. The percentage GYR varied among the parents and progenies.

Table 2 Mean square values and significant tests for individual and combined ANOVA of nine phenotypic traits of twenty-eight $F_{2}$ progenies and their eight parents evaluated under moisture stressed and non-stressed environments across two locations in Zimbabwe 


\begin{tabular}{|c|c|c|c|c|c|c|c|c|c|c|}
\hline \multirow[t]{2}{*}{ Trait } & \multicolumn{2}{|c|}{ Genotype (G) } & \multicolumn{2}{|l|}{ Location (L) } & \multirow[t]{2}{*}{ WR } & \multirow[t]{2}{*}{$G \times W R$} & \multirow[t]{2}{*}{$L \times W R$} & \multirow[t]{2}{*}{$G \times L$} & \multirow[t]{2}{*}{ GxLxWR } & \multirow{2}{*}{ Residual } \\
\hline & DS & NS & DS & NS & & & & & & \\
\hline $\begin{array}{l}\mathrm{PH} \\
(\mathrm{cm})\end{array}$ & 425.50 & $521.70^{\star \star \star}$ & $22383.10^{\star \star \star}$ & 245.90 & $17669.60^{\star \star \star}$ & 287.10 & $13660.50^{\star \star \star}$ & 276.00 & $731.30^{\star \star \star}$ & 252.10 \\
\hline DFW & $101.90^{\star \star \star}$ & $144.70^{\star \star \star}$ & 58.78 & $308.69^{\star \star \star}$ & $198.60^{\star \star \star}$ & 13.10 & $318.40^{\star \star \star}$ & 12.99 & 9.48 & 18.18 \\
\hline DPM & $97.50^{\text {** }}$ & $123.65^{\star \star \star}$ & 90.78 & $223.30^{\star \star \star}$ & $2996.70^{\star \star \star}$ & 12.03 & 14.67 & 14.97 & 12.03 & 20.94 \\
\hline DSF & $44.79^{*}$ & $6.17^{\star \star}$ & $289.95^{\star \star \star}$ & 6.89 & $1661.90^{\star \star \star}$ & $24.72^{*}$ & $193.10^{\star \star \star}$ & 11.97 & 11.95 & 15.32 \\
\hline NPPP & $395.30^{\star *}$ & $180.26^{\star \star \star}$ & $2629.40^{\star \star \star}$ & $1277.57^{\star \star \star}$ & $2308.70^{\star \star \star}$ & 148.90 & 120.70 & 85.00 & 53.90 & 137.20 \\
\hline NSP & $3.65^{\star}$ & $5.93^{* \star \star}$ & $24.04^{\star \star \star}$ & $25.00^{* \star *}$ & $10.44^{*}$ & 0.81 & $49.03^{* \star *}$ & $3.44^{\star *}$ & $3.26^{*}$ & 1.92 \\
\hline NSPP & $2415.00^{*}$ & 1385 & 195.00 & $101375^{\star \star \star}$ & 2.00 & 695.00 & $46341.00^{\star \star \star}$ & 865.00 & $2068.00^{*}$ & 1269.00 \\
\hline $\begin{array}{l}\text { GYD } \\
\text { (g) }\end{array}$ & $72486^{\star \star \star}$ & $147308^{* \star}$ & $276234^{\star \star *}$ & $1061673^{\star \star \star}$ & $73358897^{\star \star \star}$ & $66699^{*}$ & 127409.00 & 29424.00 & 25314.00 & 45543.00 \\
\hline SW (g) & $279.08^{\star \star \star}$ & $198.55^{\star \star \star}$ & 101.34 & 1.23 & $391.69^{\star \star}$ & $94.72^{\star *}$ & 40.10 & 37.47 & 36.89 & 51.92 \\
\hline$d f$ & 35 & 35 & 1 & 1 & 1 & 35 & 1 & 35 & 35 & 143 \\
\hline
\end{tabular}

$G$ genotype, $L$ location, WR water regime (environment), $G \times$ WR genotype by water regime, $L \times$ WR location by water regime, $G \times L \times W R$ genotype by location by water regime, $H^{2}$ heritability in broad sense, DS moisture stressed environments, NS non-stressed environments, $P H$ plant height, DFW days to flowering, DPM days to physiological maturity, DSF days to seed fill, NPPP number of pods per plant, NSP number of seeds per pod, NSPP number of seeds per plant, GYD grain yield, $S W 100$ seed weight, DF degrees of freedom; ${ }^{*} p<0.05,{ }^{*} p<0.01$ and ${ }^{* \star *} p<0.001$

Cross G4 X G5 had the lowest GYR of 31.29\% under DS, followed by G2 X G3 (35.56\%) among the crosses. On the other hand, cross G1 X G2 exhibited a large GYR of $56.65 \%$ under DS followed by G7 X G8 (56.26\%) among the crosses. Crosses such as G2 X G3 (0.39), G4 X G5 (0.43), and G6 X G8 (0.37) had moderate to high drought tolerance index (DTI) values. Among the parents, G6 (32.98\%) was the least sensitive to DS and exhibited the highest DTI (0.42).

Under DS, the best performing progeny (G6 X G8) with respect to GYD, SW and DPM ranked seventh in terms of NPPP (29.50), however, this was not significantly different from the NPPP of the top performer with 37.00 ( $G 1 X$ G5). This progeny also ranked second and third best with respect to GYD (2616 g) and SW (32.75 g), however, these were not significantly different from the GYD ( $2580 \mathrm{~g} ; \mathrm{G} 4 \mathrm{X} \mathrm{G8})$ and SW (41.50 g; G3 X G8) of the top performers. The progeny G4 X G8 ranked second best in SW under both DS ( $48.25 \mathrm{~g})$ and NS ( $40.25 \mathrm{~g})$, however, the SW was not significantly different from the SW of the top performers. Among the parents under DS, the best performing genotypes with respect to GYD, NPPP, NSP, NSPP and SW were G3, G6, G7, and G8. Under NS, the parent G8 ranked best for DPM, GYD, and SW and second for GYD under DS. Generally, the parents G3, G7, G4, and G8 were the top performing genotypes in terms of NSP, NSPP, GYD, SW and NPPP under NS.

Combining ability analysis of $\mathrm{F}_{2}$ progenies and their parents for grain yield and yield attributing traits under moisture stressed and non-moisture stressed conditions

Analysis of variance on combining ability revealed significant $(p<0.05)$ means square of GCA effects on DFW, DPM, DSF and SW under both DS and NS conditions (Table 4). Grain yield and NSPP had significant $(p<0.05)$ GCA effects only under NS and DS conditions respectively, while NPPP and NSP had non-significant $(p>0.05)$ GCA effects under both test conditions. Mean squares due to SCA effects were only significant $(p<0.05)$ for DFW, DPM, NPPP, GYD, and SW under both test conditions. Genotypes and SCA significantly $(p<0.01)$ interacted with location under both environments with respect to $\mathrm{PH}$, and NSP had highly significant $(p<0.001)$ location $\times \mathrm{GCA}(\mathrm{L} \times \mathrm{GCA})$ and location $\times S C A(L \times S C A)$ interactions under NS conditions.

Table 3 Mean values for grain yield (grams per 10 plants) and yield attributing traits of three key parents (based on GYD performance of their progenies) and their $\mathrm{F}_{2}$ progenies evaluated under moisture stressed and non-stressed conditions across two locations in Zimbabwe 


\begin{tabular}{|c|c|c|c|c|c|c|c|c|c|c|}
\hline \multirow[t]{2}{*}{ Genotype } & \multicolumn{2}{|c|}{$\mathrm{PH}(\mathrm{cm})$} & \multicolumn{2}{|c|}{ DFW (days) } & \multicolumn{2}{|c|}{ DPM (days) } & \multicolumn{2}{|c|}{ DSF (days) } & \multicolumn{2}{|l|}{ NPPP } \\
\hline & DS & NS & DS & NS & DS & NS & DS & NS & DS & NS \\
\hline \multicolumn{11}{|l|}{ Parent } \\
\hline G3 & 54.20 & $66.00^{a-f}$ & $56.50^{a-d}$ & $57.75^{\mathrm{a}-\mathrm{e}}$ & $85.75^{a b}$ & $92.25^{\mathrm{a}-\mathrm{e}}$ & $29.25^{\mathrm{a}-\mathrm{e}}$ & $34.50^{a-d}$ & $9.50^{a-d}$ & $27.00^{a-h}$ \\
\hline G4 & 68.20 & $78.25^{c-f}$ & $58.75^{a-g}$ & $59.00^{a-g}$ & $86.00^{\mathrm{ab}}$ & $93.75^{a-g}$ & $27.25^{\mathrm{a}-\mathrm{e}}$ & $34.75^{\mathrm{a}-\mathrm{e}}$ & $11.50^{\mathrm{a}-\mathrm{e}}$ & $2012^{a-f}$ \\
\hline G8 & 39.50 & $70.25^{b-f}$ & $58.25^{a-f}$ & $53.25^{\mathrm{ab}}$ & $84.25^{\mathrm{a}}$ & $89.50^{a}$ & $26.00^{\mathrm{a}-\mathrm{c}}$ & $36.25^{\text {de }}$ & $15.00^{\mathrm{a}-\mathrm{f}}$ & $22.38^{\mathrm{a}-\mathrm{g}}$ \\
\hline \multicolumn{11}{|c|}{$F_{2}$ Progenies } \\
\hline G1 X G3 & 62.00 & $75.46^{b-f}$ & $63.92^{\mathrm{d}-\mathrm{I}}$ & $70.21^{\mathrm{k}-\mathrm{o}}$ & $92.42^{a-g}$ & $104.17^{\mathrm{k}-\mathrm{o}}$ & $28.50^{\mathrm{a}-\mathrm{e}}$ & $33.96^{a-d}$ & $22.00^{a-f}$ & $25.94^{a-h}$ \\
\hline G1 X G4 & 56.00 & $75.25^{b-f}$ & $64.00^{\mathrm{d}-\mathrm{I}}$ & $65.25^{\mathrm{g}-\mathrm{m}}$ & $88.50^{\mathrm{a}-\mathrm{e}}$ & $97.75^{\mathrm{e}-\mathrm{j}}$ & $24.50^{a}$ & $32.50^{\mathrm{ab}}$ & $15.75^{a-f}$ & $20.50^{a-f}$ \\
\hline G1 X G8 & 53.00 & $64.00^{a-f}$ & $64.50^{\mathrm{e}-1}$ & $63.25^{\mathrm{e}-\mathrm{j}}$ & $88.75^{a-e}$ & $98.50^{f-k}$ & $24.25^{\mathrm{a}}$ & $35.25^{\mathrm{a}-\mathrm{e}}$ & $16.50^{\mathrm{a}-\mathrm{f}}$ & $26.12^{a-h}$ \\
\hline $\mathrm{G} 2 \times \mathrm{G} 3$ & 49.50 & $76.00^{b-f}$ & $57.50^{\mathrm{a}-\mathrm{e}}$ & $60.50^{c-h}$ & $88.75^{a-e}$ & $93.00^{a-f}$ & $31.25^{\mathrm{a}-\mathrm{e}}$ & $32.50^{a b}$ & $18.00^{a-f}$ & $37.88^{g h}$ \\
\hline $\mathrm{G} 2 \times \mathrm{G} 4$ & 45.20 & $61.75^{a-e}$ & $62.50^{c-k}$ & $61.75^{\mathrm{d}-\mathrm{i}}$ & $93.50^{\mathrm{b}-\mathrm{g}}$ & $96.25^{\mathrm{b}-\mathrm{i}}$ & $31.00^{a-e}$ & $34.50^{a-d}$ & $14.00^{a-f}$ & $25.00^{a-h}$ \\
\hline $\mathrm{G} 2 \times \mathrm{G} 8$ & 59.00 & $56.25^{a-d}$ & $63.75^{\mathrm{d}-\mathrm{I}}$ & $65.50^{\mathrm{g}-\mathrm{m}}$ & $89.75^{\mathrm{a}-\mathrm{e}}$ & $98.50^{f-k}$ & $26.00^{\mathrm{a}-\mathrm{c}}$ & $33.00^{\mathrm{a}-\mathrm{c}}$ & $24.00^{\mathrm{a}-\mathrm{f}}$ & $31.38^{d-h}$ \\
\hline $\mathrm{G} 3 \times \mathrm{G} 4$ & 43.00 & $59.50^{\mathrm{a}-\mathrm{e}}$ & $56.75^{a-d}$ & $58.50^{a-f}$ & $88.25^{\mathrm{a}-\mathrm{e}}$ & $91.75^{a-d}$ & $31.50^{\mathrm{a}-\mathrm{e}}$ & $33.25^{\mathrm{a}-\mathrm{d}}$ & $8.00^{a-c}$ & $14.50^{a-c}$ \\
\hline G3 X G5 & 79.20 & $82.75^{\mathrm{d}-\mathrm{f}}$ & $65.75^{f-m}$ & $69.00^{\mathrm{j}-\mathrm{o}}$ & $95.50^{c-i}$ & $102.75^{\mathrm{j}-\mathrm{m}}$ & $29.75^{\mathrm{a}-\mathrm{e}}$ & $33.75^{a-d}$ & $33.00^{\mathrm{ef}}$ & $28.50^{\mathrm{c}-\mathrm{h}}$ \\
\hline G3 X G6 & 51.20 & $60.17^{\mathrm{a}-\mathrm{e}}$ & $61.92^{c-k}$ & $61.50^{d-i}$ & $92.33^{a-f}$ & $95.08^{a-h}$ & $30.67^{a-e}$ & $34.08^{a-d}$ & $10.50^{\mathrm{a}-\mathrm{e}}$ & $19.83^{a-f}$ \\
\hline G3 X G7 & 49.20 & $56.25^{a-d}$ & $62.25^{c-k}$ & $64.75^{f-m}$ & $92.75^{a-g}$ & $100.50^{\mathrm{h}-\mathrm{I}}$ & $30.50^{\mathrm{a}-\mathrm{e}}$ & $35.75^{c-e}$ & $36.00^{f}$ & $30.00^{c-h}$ \\
\hline G3 X G8 & 50.50 & $53.00^{\mathrm{a}-\mathrm{c}}$ & $55.00^{\mathrm{a}-\mathrm{c}}$ & $52.75^{a}$ & $86.75^{a-c}$ & $90.50^{\mathrm{ab}}$ & $31.75^{\mathrm{a}-\mathrm{e}}$ & $37.75^{\mathrm{e}}$ & $31.50^{\mathrm{d}-\mathrm{f}}$ & $29.25^{c-h}$ \\
\hline G4 X G5 & 48.20 & $64.25^{a-f}$ & $60.75^{\mathrm{b}-\mathrm{j}}$ & $62.75^{\mathrm{e}-\mathrm{j}}$ & $93.25^{b-g}$ & $96.50^{c-i}$ & $32.50^{\mathrm{a}-\mathrm{e}}$ & $33.75^{a-d}$ & $12.75^{\mathrm{a}-\mathrm{e}}$ & $39.12^{\mathrm{h}}$ \\
\hline G4 X G6 & 54.70 & $72.08^{b-f}$ & $60.17^{a-i}$ & $60.08^{c-h}$ & $88.42^{\mathrm{a}-\mathrm{e}}$ & $95.17^{a-h}$ & $28.50^{\mathrm{a}-\mathrm{e}}$ & $35.58^{\mathrm{b}-\mathrm{e}}$ & $23.92^{a-f}$ & $27.87^{b-h}$ \\
\hline G4 X G7 & 40.50 & $71.00^{\mathrm{b}-f}$ & $57.50^{\mathrm{a}-\mathrm{e}}$ & $63.75^{\mathrm{e}^{-k}}$ & $93.75^{\mathrm{b}-\mathrm{g}}$ & $98.50^{f-k}$ & $36.25^{\mathrm{e}}$ & $34.75^{\mathrm{a}-\mathrm{e}}$ & $14.50^{\mathrm{a}-\mathrm{f}}$ & $15.25^{a-d}$ \\
\hline G4 X G8 & 64.00 & $84.50^{d-f}$ & $53.25^{\mathrm{a}}$ & $54.50^{\mathrm{a}-\mathrm{c}}$ & $88.50^{\mathrm{a}-\mathrm{e}}$ & $90.75^{a-c}$ & $35.25^{\mathrm{d}-\mathrm{e}}$ & $36.25^{\text {de }}$ & $36.25^{f}$ & $36.12^{f-h}$ \\
\hline G5 X G8 & 50.70 & $84.25^{\mathrm{d}-\mathrm{f}}$ & $66.00^{\mathrm{g}-\mathrm{m}}$ & $67.50^{\mathrm{i}-\mathrm{m}}$ & $95.00^{c-h}$ & $101.50^{i-m}$ & $29.00^{a-e}$ & $34.00^{a-d}$ & $7.75^{a-c}$ & $11.62^{\mathrm{ab}}$ \\
\hline G6 X G8 & 61.50 & $62.50^{\mathrm{a}-\mathrm{e}}$ & $54.25^{\mathrm{ab}}$ & $55.50^{a-d}$ & $86.75^{a-c}$ & $91.00^{a-d}$ & $32.50^{\mathrm{a}-\mathrm{e}}$ & $35.50^{\mathrm{b}-\mathrm{e}}$ & $29.50^{b-f}$ & $24.88^{a-h}$ \\
\hline G7 X G8 & 62.70 & $66.25^{a-f}$ & $62.50^{\mathrm{c}-k}$ & $62.75^{\mathrm{e}-\mathrm{j}}$ & $89.50^{a-e}$ & $97.75^{\mathrm{e}-\mathrm{j}}$ & $27.00^{a-d}$ & $35.00^{a-e}$ & $24.00^{a-f}$ & $26.25^{a-h}$ \\
\hline Mean & 54.30 & 70.00 & 62.67 & 64.33 & 92.33 & 98.78 & 29.68 & 34.48 & 18.80 & 24.45 \\
\hline SED & 17.52 & 11.44 & 3.11 & 2.79 & 3.56 & 2.44 & 3.64 & 1.28 & 9.34 & 6.57 \\
\hline LSD (5\%) & $\mathrm{ns}$ & 22.68 & 6.17 & 5.52 & 7.05 & 4.83 & 7.22 & 2.53 & 18.52 & 13.02 \\
\hline
\end{tabular}

Table 3 continued 


\begin{tabular}{|c|c|c|c|c|c|c|c|c|c|c|}
\hline \multirow{2}{*}{ Genotype } & \multicolumn{2}{|l|}{ NSP } & \multicolumn{2}{|l|}{ NSPP } & \multicolumn{2}{|l|}{ GYD (g) } & \multicolumn{2}{|l|}{ SW (g) } & \multirow[t]{2}{*}{$\%$ GYR } & \multirow[t]{2}{*}{ DTI } \\
\hline & DS & NS & DS & NS & DS & NS & DS & NS & & \\
\hline \multicolumn{11}{|l|}{ Parent } \\
\hline G3 & $7.00^{b-d}$ & $7.50^{a-d}$ & $48.00^{a-d}$ & 74.50 & $1153.00^{\mathrm{b}-\mathrm{j}}$ & $2010.00^{a-d}$ & $15.75^{a-c}$ & $25.50^{a-f}$ & 42.62 & 0.31 \\
\hline G4 & $6.00^{a-c}$ & $7.25^{a-d}$ & $40.00^{a-d}$ & 50.00 & $1071.00^{\mathrm{a}-\mathrm{g}}$ & $2051.00^{\mathrm{a}-\mathrm{d}}$ & $26.50^{d-h}$ & $41.00^{\mathrm{h}}$ & 47.79 & 0.26 \\
\hline G8 & $6.25^{a-c}$ & $6.50^{a-d}$ & $57.75^{\mathrm{a}-\mathrm{d}}$ & 42.30 & $1188.00^{c-j}$ & $2362.00^{\mathrm{b}-f}$ & $28.00^{\mathrm{e}-\mathrm{h}}$ & $30.25^{d-h}$ & 49.71 & 0.28 \\
\hline \multicolumn{11}{|c|}{$F_{2}$ Progenies } \\
\hline G1 X G3 & $6.46^{a-c}$ & $6.42^{\mathrm{a}-\mathrm{d}}$ & $75.21^{\mathrm{a}-\mathrm{e}}$ & 67.00 & $1040.00^{a-f}$ & $2129.00^{\mathrm{a}-\mathrm{e}}$ & $14.83^{a-c}$ & $21.00^{a-f}$ & 51.16 & 0.24 \\
\hline G1 X G4 & $6.75^{a-d}$ & $6.75^{a-d}$ & $64.25^{\mathrm{a}-\mathrm{d}}$ & 60.50 & $956.00^{a-c}$ & $2116.00^{\mathrm{a}-\mathrm{d}}$ & $15.50^{\mathrm{a}-\mathrm{c}}$ & $17.25^{a-d}$ & 54.79 & 0.20 \\
\hline G1 X G8 & $6.75^{a-d}$ & $6.75^{a-d}$ & $54.75^{\mathrm{a}-\mathrm{d}}$ & 62.00 & $1243.00^{\mathrm{e}-\mathrm{k}}$ & $2122.00^{\mathrm{a}-\mathrm{e}}$ & $20.75^{a-g}$ & $25.75^{a-f}$ & 41.45 & 0.34 \\
\hline G2 X G3 & $9.25^{d}$ & $11.25^{\mathrm{e}}$ & $68.00^{a-d}$ & 58.50 & $1300.00^{g-k}$ & $2018.00^{a-d}$ & $18.50^{\mathrm{a}-\mathrm{e}}$ & $23.50^{a-f}$ & 35.56 & 0.39 \\
\hline $\mathrm{G} 2 \times \mathrm{G} 4$ & $6.50^{a-c}$ & $7.25^{\mathrm{a}-\mathrm{d}}$ & $64.00^{\mathrm{a}-\mathrm{d}}$ & 72.50 & $1241.00^{\mathrm{e}-\mathrm{k}}$ & $2060.00^{a-d}$ & $18.75^{\mathrm{a}-\mathrm{e}}$ & $20.75^{a-f}$ & 39.73 & 0.35 \\
\hline G2 X G8 & $7.00^{b-d}$ & $6.75^{\mathrm{a}-\mathrm{d}}$ & $61.25^{\mathrm{a}-\mathrm{d}}$ & 77.00 & $1373.00^{j-k}$ & $2240.00^{a-f}$ & $23.50^{\mathrm{ch} h}$ & $23.50^{a-f}$ & 38.71 & 0.40 \\
\hline G3 X G4 & $6.00^{a-c}$ & $7.00^{\mathrm{a}-\mathrm{d}}$ & $37.00^{a-c}$ & 47.00 & $1082.00^{a-h}$ & $1982.00^{a-c}$ & $22.25^{\mathrm{b}-\mathrm{h}}$ & $21.50^{a-f}$ & 45.42 & 0.28 \\
\hline G3 X G5 & $8.00^{\mathrm{cd}}$ & $8.00^{b-d}$ & $51.00^{a-d}$ & 64.20 & $1024.00^{a-f}$ & $2115.00^{\mathrm{a}-\mathrm{d}}$ & $19.50^{\mathrm{a}-\mathrm{f}}$ & $18.50^{a-d}$ & 51.60 & 0.23 \\
\hline G3 X G6 & $6.83^{a-d}$ & $7.67^{a-d}$ & $44.00^{a-d}$ & 54.90 & $1092.00^{a-i}$ & $2000.00^{\mathrm{a}-\mathrm{d}}$ & $22.08^{b-h}$ & $19.83^{a-e}$ & 45.44 & 0.28 \\
\hline G3 X G7 & $7.25^{b-d}$ & $7.75^{a-d}$ & $89.50^{\mathrm{b}-\mathrm{e}}$ & 102.30 & $1117^{a-i}$ & $2293.00^{a-f}$ & $17.75^{\mathrm{a}-\mathrm{d}}$ & $16.00^{\mathrm{ab}}$ & 51.29 & 0.26 \\
\hline G3 X G8 & $5.75^{a-c}$ & $6.25^{a-d}$ & $42.50^{\mathrm{a}-\mathrm{d}}$ & 55.00 & $1187.00^{c-j}$ & $2375.00^{c-f}$ & $28.75^{f-h}$ & $41.50^{\mathrm{h}}$ & 50.04 & 0.28 \\
\hline G4 X G5 & $6.50^{a-c}$ & $6.50^{\mathrm{a}-\mathrm{d}}$ & $56.25^{\mathrm{a}-\mathrm{d}}$ & 60.00 & $1326.00^{i-k}$ & $1930.00^{a-c}$ & $19.50^{\mathrm{a}-\mathrm{f}}$ & $17.50^{a-d}$ & 31.29 & 0.43 \\
\hline G4 X G6 & $7.08^{b-d}$ & $8.25^{\mathrm{cd}}$ & $53.42^{\mathrm{a}-\mathrm{d}}$ & 65.90 & $1170.00^{c-j}$ & $2236.00^{a-f}$ & $30.83^{h}$ & $28.08^{\mathrm{b}-\mathrm{g}}$ & 47.68 & 0.29 \\
\hline G4 X G7 & $6.50^{a-c}$ & $7.50^{a-d}$ & $30.50^{\mathrm{a}-\mathrm{c}}$ & 41.00 & $994.00^{a-d}$ & $2062.00^{\mathrm{a}-\mathrm{d}}$ & $29.25^{\mathrm{gh}}$ & $22.00^{a-f}$ & 51.83 & 0.23 \\
\hline G4 X G8 & $6.50^{a-c}$ & $7.25^{\mathrm{a}-\mathrm{d}}$ & $87.75^{\mathrm{b}-\mathrm{e}}$ & 88.50 & $1256.00^{f-k}$ & $2580.00^{e f}$ & $48.25^{i}$ & $40.25^{\mathrm{gh}}$ & 51.31 & 0.29 \\
\hline G5 X G8 & $5.00^{\mathrm{ab}}$ & $5.50^{a-c}$ & $22.50^{\mathrm{ab}}$ & 14.00 & $924.00^{\mathrm{ab}}$ & $1892.00^{\mathrm{ab}}$ & $14.45^{\mathrm{a}-\mathrm{c}}$ & $20.50^{a-f}$ & 51.15 & 0.21 \\
\hline G6 X G8 & $5.00^{\mathrm{ab}}$ & $5.00^{\mathrm{a}}$ & $61.50^{\mathrm{a}-\mathrm{d}}$ & 60.30 & $1428.00^{k}$ & $2616.00^{f}$ & $50.25^{i}$ & $32.75^{e-h}$ & 45.43 & 0.37 \\
\hline G7 X G8 & $7.50^{b-d}$ & $7.50^{a-d}$ & $57.50^{\mathrm{a}-\mathrm{d}}$ & 72.00 & $1077.00^{\mathrm{a}-\mathrm{h}}$ & $2462.00^{d-f}$ & $22.50^{\mathrm{b}-\mathrm{h}}$ & $26.00^{a-f}$ & 56.26 & 0.22 \\
\hline Mean & 6.41 & 6.79 & 59.90 & 60.00 & 1116.00 & 2125.00 & 21.64 & 23.98 & 47.32 & \\
\hline SED & 1.09 & 1.19 & 27.80 & 32.03 & 99.50 & 189.00 & 3.97 & 5.61 & & \\
\hline LSD (5\%) & 2.16 & 2.36 & 55.11 & ns & 197.30 & 374.70 & 7.87 & 11.13 & & \\
\hline
\end{tabular}

PH plant height, DFW days to flowering, DPM days to physiological maturity, DSF days to seed fill, NPPP number of pods per plant, NSP number of seeds per pod, NSPP number of seeds per plant, GYD grain yield, SW100 seed weight, DS moisture stressed environments, NS non-moisture stressed environments, GYR percentage grain yield reduction, DT/ drought tolerance index, SED standard error of differences, $L S D$ least significant difference at $0.05,{ }^{*} p<0.05,{ }^{*} p<0.01,{ }^{* *} p<0.001$, Means in a column followed by the same letter(s) are not significantly different at $p \geq 0.05$ under DS and NS conditions.

On the other hand, GCA and SCA significantly $(p<0.05)$ interacted with location under DS environment with respect to DFW and NSPP, respectively. Under NS, all the Baker's ratios for the characters studied were more than 0.5 but less than unity. In addition, under NS, the estimate of GCA variance was relatively greater than that of SCA for all the studied traits except for PH, and NPPP as revealed by ratios of GCA: SCA variances. However, under DS environments, all the Baker's genetic ratios for the studied traits were more than 0.5 except for NPPP. Furthermore, under DS, the estimate of GCA: SCA ratio was relatively greater (more than 1 ) than that of SCA for all traits except for PH, NPPP and NSP.

General combining ability effects of parental lines for grain yield and yield attributing traits under moisture stressed environment 
Estimates of the GCA effects for the eight parents under DS conditions are presented in Table 5. Parents which combine significant, low, and negative GCA effects for DFW, DSF, and DPM with significant, positive, and high GCA effects for GYD are considered desirable under DS environments. On the other hand, parents that exhibit significant and positive GCA effects for NPPP, NSP, NSPP, GYD, and SW are preferred for improving these traits under DS conditions. Significant $(p<0.01)$ positive and desirable GCA effects for GYD were observed on $\mathrm{G} 6$ and $\mathrm{G} 8$, while $\mathrm{G} 3$ had significant $(p<0.01)$ positive GCA effects for NSP. Notably, G8 had the highest positive, desirable and significant $(p<0.01)$ GCA effects for GYD under moisture stressed conditions, followed by G6. Thus, G8 and G6 were the best combiners for GYD under DS. Additionally, parents G4, G6 and G8 showed consistent desirable positive GCA effects for SW. Significant $(p<0.05)$ and negative GCA effects for GYD, NSP, SW, DPM, and NSPP were observed on parent G5 under DS. G1, G6, and G7 were high general combiners for NSPP, G6 for DSF, G3 for NSP, G1 for NPPP, PH and DFW.

General combining ability effects of parental lines for grain yield and yield attributing traits under non-moisture stressed environment

Estimates of the GCA effects for the eight parents under NS conditions are presented in Table 5. Parents that exhibit significant and positive GCA effects for NPPP, NSP, NSPP, GYD, and SW are preferred for improving these traits under NS conditions.

Table 4 Analysis of variance, combining ability effects, GCA: SCA ratio and Baker's ratio for grain yield and yield-attributing traits under moisture stressed and non-moisture stressed environments across two locations in Zimbabwe 


\begin{tabular}{|c|c|c|c|c|c|c|c|c|c|c|}
\hline \multicolumn{11}{|c|}{ Mean squares } \\
\hline $\begin{array}{l}\text { Source of } \\
\text { Variation } d\end{array}$ & & $\mathrm{PH}(\mathrm{cm})$ & DFW & DPM & $\begin{array}{l}\text { DSF } \\
\text { (days) }\end{array}$ & NPPP & NSP & NSPP & GYD (g) & SW (g) \\
\hline \multicolumn{11}{|c|}{ Moisture stressed environments } \\
\hline $\begin{array}{l}\text { Location } \\
\text { (Loc) }\end{array}$ & 1 & $5121.24^{\star \star \star}$ & 20.70 & 66.81 & $289.94^{\star \star \star}$ & $1104.96^{\star \star}$ & $15.23^{\star \star}$ & 85.09 & 175603.60 ** & 101.33 \\
\hline $\begin{array}{l}\text { Replication } \\
\text { (Loc) }\end{array}$ & 2 & 84.57 & $76.48^{\star \star \star}$ & 516.3 & $141.69^{\star \star}$ & 87.35 & $7.34^{\star \star \star}$ & 113.49 & 264.69 & 62.34 \\
\hline $\begin{array}{l}\text { Genotype } \\
\text { (G) }\end{array}$ & 35 & 405.02 & $86.07^{\star \star \star}$ & $90.76^{\star \star \star}$ & $44.79^{*}$ & $347.31^{\star \star \star}$ & $3.52^{\star \star \star}$ & 2091.44 & $65374.65^{\star \star \star}$ & $279.08^{\star \star \star}$ \\
\hline GCA & 7 & 427.89 & $293.59^{\star \star \star}$ & $275.78^{\star \star \star}$ & $64.07^{*}$ & 175.93 & 3.47 & $2306.10^{\star \star \star}$ & 100339.70 & $672.53^{\star \star}$ \\
\hline SCA & 28 & 459.38 & $47.86^{\star \star \star}$ & $51.10^{\star \star}$ & 39.97 & $430.28^{\star \star \star}$ & $3.84^{*}$ & 2297.89 & $63208.22^{\star \star \star}$ & $180.72^{\star \star \star}$ \\
\hline Loc X G & 35 & $510.07^{\star *}$ & 13.32 & 19.80 & 22.20 & $86.78^{\star \star \star}$ & 1.72 & 1853.56 & 2671.10 & 26.37 \\
\hline Loc X GCA & 7 & 781.07 & $28.02^{\star}$ & 29.07 & 14.23 & 87.46 & 2.56 & 284.36 & 3329.16 & 40.25 \\
\hline Loc X SCA & 28 & $547.19^{\star \star}$ & 12.15 & 19.10 & 24.19 & 94.26 & 1.81 & $2388.91^{*}$ & 2557.36 & 22.90 \\
\hline Residual & 50 & 218.74 & 14.87 & 20.22 & 24.58 & 146.25 & 1.88 & 1167.55 & 23072.69 & 32.14 \\
\hline GCA:SCA & & 0.93 & 6.13 & 5.36 & 1.60 & 0.41 & 0.90 & 1.00 & 1.59 & 3.72 \\
\hline $\begin{array}{l}\text { Baker's } \\
\text { ratio }\end{array}$ & & 0.65 & 0.92 & 0.92 & 0.76 & 0.45 & 0.64 & 0.67 & 0.76 & 0.88 \\
\hline \multicolumn{11}{|c|}{ Non-moisture stressed environments } \\
\hline $\begin{array}{l}\text { Location } \\
\text { (Loc) }\end{array}$ & 1 & 92.34 & $107.30^{\star *}$ & $86.28^{\star \star}$ & 6.89 & $693.34^{\star *}$ & $9.21^{*}$ & $57673.36^{\star \star \star}$ & $945854.17^{\star \star \star}$ & 0.67 \\
\hline $\begin{array}{l}\text { Replication } \\
\text { (Loc) }\end{array}$ & 2 & 3.88 & $15.19^{\star}$ & $13.98^{*}$ & 3.97 & $51.36^{*}$ & 0.62 & $597.03^{*}$ & $47016.58^{\star \star \star}$ & $85.92^{\star \star \star}$ \\
\hline $\begin{array}{l}\text { Genotype } \\
\text { (G) }\end{array}$ & 35 & 453.41 & $120.83^{\star \star \star}$ & $107.45^{\star \star \star}$ & $6.16^{\star * \star}$ & $164.38^{\star \star \star}$ & 5.17 & 1316.66 & $142123.88^{\star \star \star}$ & $196.74^{\star \star \star}$ \\
\hline GCA & 7 & $337.99^{*}$ & $477.54^{\star \star \star}$ & $387.76^{* \star \star}$ & $10.80^{*}$ & 105.23 & 6.06 & 2305.74 & $334959.49^{*}$ & $374.95^{*}$ \\
\hline SCA & 28 & 530.13 & $55.53^{\star \star \star}$ & $51.94^{\star \star \star}$ & $5.00^{* *}$ & $198.81^{\star * *}$ & 5.43 & 1189.80 & $100419.46^{*}$ & $166.06^{* \star *}$ \\
\hline Loc X G & 35 & $418.21^{\star \star \star}$ & 6.96 & 6.33 & 1.73 & 30.53 & $4.22^{\star \star \star}$ & 982.26 & 50336.72 & 41.13 \\
\hline Loc X GCA & 7 & 122.63 & 10.15 & 9.69 & 0.76 & 19.48 & 5.61 & 998.93 & 64448.15 & 40.43 \\
\hline Loc X SCA & 28 & $505.72^{\star \star \star}$ & 7.17 & 6.23 & 1.97 & 36.17 & $4.82^{\star \star \star}$ & 1061.95 & 47985.91 & 46.81 \\
\hline Residual & 50 & 146.58 & 12.41 & 9.41 & 3.92 & 86.35 & 1.38 & 1053.44 & 65394.42 & 62.12 \\
\hline GCA:SCA & & 0.64 & 8.60 & 7.47 & 2.16 & 0.53 & 1.12 & 1.94 & 3.34 & 2.26 \\
\hline $\begin{array}{l}\text { Baker's } \\
\text { ratio }\end{array}$ & 1 & 0.56 & 0.95 & 0.94 & 0.81 & 0.51 & 0.69 & 0.79 & 0.87 & 0.82 \\
\hline
\end{tabular}

$D F W$ days to flowering DPM days to physiological maturity, DSF days to seed fill, NPPP number of pods per plant, NSPP number of seeds per plant, $N S P$ number of seed per pod, GYD grain yield, SW 100 seed weight, $d f$ degrees of freedom, GCA general combining ability, SCA specific combining ability, ${ }^{*} p<0.05,{ }^{* *} p<0.01,{ }^{* * *} p<0.001$

The parent G8 was the best general combiner for GYD as revealed by its significant $(p<0.001)$, positive and high GCA effects, followed by G6. The same trend was realized under DS conditions. Therefore, both G8 and G6 would be good general combiners for grain yield under DS and NS conditions. On the other hand, significant $(p<0.05)$ and negative GCA effects for GYD were observed on parents G2 and G5. G1 and G7 were high general combiners for NSPP with significant $(p<0.05)$ and positive GCA effects, $\mathrm{G} 4$ and G8 for SW with significant $(p<0.05)$ positive GCA effects, and G4 for NSP with significant $(p<0.05)$ positive GCA effects.

Specific combining ability estimates for grain yield and yield attributing traits under moisture stressed conditions 
The estimates of SCA of $28 \mathrm{~F}_{2}$ families for GYD and its components are presented in Table 6 and Online Resource 3. Four crosses, namely G3 X G5, G3 X G7, G4 X G8, and G1 X G5 exhibited significant $(p<0.05)$ and positive SCA effects for NPPP under DS conditions. On the other hand, four crosses, namely G4 X G5, G2 X G8, G2 X G3, and G6 X G8 showed significant $(p<0.05)$ and positive SCA effects for GYD. However, significant $(p<$ 0.05 ) and negative SCA effects on GYD were exhibited by $\mathrm{G} 2$ X G6 and G5 X G8. For DPM, significant $(p<0.05)$ negative SCA effects were exhibited by $\mathrm{G} 1 \times$ G4, G1 X G7, G4 X G6, G5 X G7, and G6 X G8. Thus, parent combination G6 X G8 combined significant $(p<0.05)$, negative and positive SCA effects for DPM and GYD respectively. Good specific combiners for NSP with significant $(p<0.05)$ SCA effects were G1 X G5, G2 X G3, and G3 X G5. Cross-combinations of G4 X G8, G5 X G7, and G6 X G8 showed significant $(p<0.05)$ and positive SCA effects for SW. Significant ( $p<0.05)$ and positive SCA effects for NSPP were exhibited by $\mathrm{G} 1$ X G2, G4 X G8, G5 X G6, and G6 X G7.

Specific combining ability estimates for grain yield and yield attributing traits under non-moisture stressed conditions

Higher positive and significant SCA values were considered desirable for GYD and its components under NS environments (Table 6). The parent combination G4 X G5 showed non-significant $(p>0.05)$ and negative SCA estimates for GYD under NS despite exhibiting significant SCA estimates for GYD under DS conditions. In addition, significant $(p<0.05)$ and negative SCA effects on GYD were exhibited by crosses $\mathrm{G} 1 \mathrm{X}$ G7 and G5 X G8.

Table 5 General combining ability estimates of parents for grain yield and yield-attributing traits under moisture-stressed and non-stressed conditions

\begin{tabular}{|c|c|c|c|c|c|c|c|c|c|c|}
\hline \multirow[t]{2}{*}{ Parents } & \multicolumn{2}{|c|}{$\mathrm{PH}(\mathrm{cm})$} & \multicolumn{2}{|c|}{ DFW (days) } & \multicolumn{2}{|c|}{ DPM (days) } & \multicolumn{2}{|c|}{ DSF (days) } & \multicolumn{2}{|l|}{ NPPP } \\
\hline & DS & NS & DS & NS & DS & NS & DS & NS & DS & NS \\
\hline $\mathrm{G} 1$ & $7.36^{* *}$ & -2.14 & $4.28^{\star \star \star}$ & $5.06^{\star \star \star}$ & $2.76^{\star \star \star}$ & $4.41^{\star \star \star}$ & $-2.06^{\star \star}$ & $-0.66^{*}$ & 2.56 & $2.88^{*}$ \\
\hline $\mathrm{G} 2$ & 0.03 & $3.39^{*}$ & 0.81 & $1.44^{\star *}$ & $1.42^{*}$ & 0.72 & 1.04 & $-0.73^{\star \star}$ & $-3.52^{*}$ & $-2.32^{*}$ \\
\hline G3 & -1.80 & $-3.76^{\star}$ & $-2.56^{\star \star \star}$ & $-2.58^{\star \star \star}$ & $-2.29^{\star \star}$ & $-2.66^{\star \star \star}$ & 0.53 & -0.03 & 1.18 & 1.16 \\
\hline G4 & -1.54 & 1.16 & $-3.26^{\star \star \star}$ & $-3.41^{\star \star \star}$ & $-2.47^{\star \star \star}$ & $-3.56^{\star \star \star}$ & 0.69 & -0.03 & -1.20 & -0.30 \\
\hline G5 & -1.39 & -0.12 & $2.11^{\star \star \star}$ & $1.93^{\star \star \star}$ & $1.31^{*}$ & $2.04^{\star \star \star}$ & -0.86 & 0.17 & -2.03 & -1.14 \\
\hline G6 & -1.27 & $4.33^{*}$ & $1.37^{\star}$ & $2.29^{\star \star \star}$ & $3.35^{\star \star \star}$ & $2.43^{\star \star \star}$ & $1.86^{\star \star}$ & 0.13 & -0.09 & -0.24 \\
\hline G7 & -3.00 & $-2.97^{*}$ & 0.22 & 0.58 & -0.30 & $0.80^{*}$ & -0.26 & 0.27 & 1.30 & -1.00 \\
\hline G8 & 1.61 & 0.11 & $-2.97^{\star \star \star}$ & $-5.32^{\star \star \star}$ & $-3.77^{\star \star \star}$ & $-4.18^{\star \star \star}$ & -0.94 & $0.89^{\star \star}$ & 1.79 & 0.96 \\
\hline Parents & NSP & & NSPP & & GYD (g) & & SW (g) & & & \\
\hline & DS & NS & DS & NS & DS & NS & DS & NS & & \\
\hline $\mathrm{G} 1$ & -0.06 & -0.27 & 8.20 & $7.90^{*}$ & -32.16 & 12.35 & $-4.60^{\star \star \star}$ & -0.68 & & \\
\hline $\mathrm{G} 2$ & -0.03 & -0.11 & -0.08 & -7.34 & -15.28 & $-89.19^{*}$ & $-3.93^{\star \star \star}$ & -1.04 & & \\
\hline G3 & $0.60^{\star *}$ & $0.71^{\star \star \star}$ & -2.06 & 3.95 & 1.60 & -19.02 & $-1.96^{\star \star}$ & -0.23 & & \\
\hline G4 & 0.02 & $0.41^{*}$ & -5.23 & -0.35 & 12.56 & -5.24 & $4.25^{\star \star \star}$ & $3.32^{\star \star \star}$ & & \\
\hline G5 & $-0.42^{*}$ & $-0.41^{*}$ & $-12.27^{\star}$ & $-12.58^{\star \star}$ & $-79.10^{\star \star}$ & $-118.37^{\star \star}$ & $-2.04^{*}$ & $-3.69^{\star \star}$ & & \\
\hline G6 & -0.10 & -0.04 & $8.80^{\star}$ & 3.90 & $55.80^{\star \star}$ & 21.84 & $2.34^{\star \star}$ & -1.02 & & \\
\hline G7 & 0.16 & 0.06 & 7.35 & $8.89^{*}$ & -22.49 & 9.08 & -1.03 & $-2.39^{\star}$ & & \\
\hline G8 & -0.17 & $-0.35^{*}$ & -4.71 & -4.36 & $79.09^{\star \star}$ & $188.55^{\star \star \star}$ & $6.97^{\star \star \star}$ & $5.71^{\star \star \star}$ & & \\
\hline
\end{tabular}

See footnote in Table 1 for genotype codes. PH plant height, DFW days to flowering, DPM days to physiological maturity, DSF days to seed fill, NPPP number of pods per plant, NSP number of seeds per pod, NSPP number of seeds per plant, GYD grain yield, $S W 100$ seed weight, $D S$ moisture stressed environments, NS non-moisture stressed environments, ${ }^{*} p<0.05,{ }^{* *} p<0.01,{ }^{* *} p<0.001$.

Table 6 Specific combining ability effects of 28 crosses for grain yield ( $\mathrm{g} / 10$ plants) and yield-attributing traits under moisture stressed and non-stressed conditions 


\begin{tabular}{|c|c|c|c|c|c|c|c|c|c|c|}
\hline \multirow[t]{2}{*}{ Cross } & \multicolumn{2}{|c|}{$\mathrm{PH}(\mathrm{cm})$} & \multicolumn{2}{|c|}{ DFW (days) } & \multicolumn{2}{|c|}{ DPM (days) } & \multicolumn{2}{|c|}{ DSF (days) } & \multicolumn{2}{|c|}{ NPPP } \\
\hline & DS & NS & DS & NS & DS & NS & DS & NS & DS & NS \\
\hline \multicolumn{11}{|c|}{ SCA effects of 10 top yielding crosses under drought stress } \\
\hline G1 X G5 & 6.50 & 10.34 & -2.98 & -2.79 & 0.63 & -1.48 & 3.74 & 1.52 & $16.16^{\star *}$ & 7.10 \\
\hline G1 X G7 & -13.30 & $-24.99^{\star \star \star}$ & $-5.00^{\star \star}$ & $-6.70^{\star \star \star}$ & $-7.10^{\star \star}$ & $-7.25^{\star \star \star}$ & -2.36 & -0.33 & $-17.31^{\star \star}$ & 2.41 \\
\hline G1 X G8 & -0.51 & -1.60 & -0.34 & -0.56 & -2.62 & -0.23 & -2.43 & 0.54 & -10.12 & -0.31 \\
\hline $\mathrm{G} 2 \times \mathrm{G} 3$ & -5.00 & 5.28 & -2.91 & -2.69 & -2.77 & -3.74 & 0.00 & -1.22 & 0.20 & $13.87^{\star \star}$ \\
\hline $\mathrm{G} 2 \times \mathrm{G} 4$ & -5.59 & $-11.66^{*}$ & 2.81 & -0.56 & 2.18 & 0.44 & -0.41 & 0.78 & 0.08 & 3.29 \\
\hline G2 X G7 & -8.57 & -2.05 & -0.96 & -1.34 & -3.02 & -1.92 & -1.96 & -0.51 & -6.27 & -2.06 \\
\hline $\mathrm{G} 2 \times \mathrm{G} 8$ & 5.81 & $-14.34^{\star \star}$ & $3.52^{*}$ & $5.53^{\star *}$ & -0.38 & $3.84^{\star \star}$ & -3.78 & -1.64 & 5.78 & 7.91 \\
\hline G4 X G5 & -3.81 & -6.53 & -0.67 & -0.70 & 2.08 & -1.42 & 2.99 & -0.87 & -2.85 & $15.59^{\star * \star}$ \\
\hline G4 X G8 & 7.80 & $13.88^{*}$ & $-4.68^{*}$ & -2.91 & 2.03 & -1.83 & $5.82^{*}$ & 0.90 & $19.71^{\star \star \star}$ & $9.77^{*}$ \\
\hline G6 X G8 & 11.68 & -8.27 & $-6.43^{\star \star \star}$ & $-5.82^{\star \star \star}$ & $-5.02^{*}$ & $-5.72^{\star \star \star}$ & 1.90 & -0.01 & 6.00 & -0.17 \\
\hline \multirow[t]{2}{*}{ Cross } & \multicolumn{3}{|l|}{ NSP } & NSPP & \multicolumn{2}{|l|}{ GYD (g) } & \multicolumn{2}{|l|}{ SW (g) } & & \\
\hline & DS & NS & DS & NS & DS & NS & DS & NS & & \\
\hline \multicolumn{11}{|c|}{ SCA effects of 10 top yielding crosses under drought stress } \\
\hline G1 XG5 & $1.41^{*}$ & $1.30^{*}$ & 26.72 & $31.21^{*}$ & 53.80 & 183.80 & 2.73 & 6.08 & & \\
\hline $\mathrm{G} 1 \times \mathrm{G} 7$ & 0.77 & 0.98 & -29.82 & 4.36 & 61.34 & $-233.66^{*}$ & -1.77 & -0.82 & & \\
\hline G1 X G8 & 0.59 & 0.77 & -13.89 & 1.48 & 103.94 & -209.77 & -3.26 & -3.60 & & \\
\hline $\mathrm{G} 2 \times \mathrm{G} 3$ & $2.23^{\star \star \star}$ & $3.53^{\star \star \star}$ & 10.55 & -0.24 & $184.80^{\star *}$ & 0.56 & 2.75 & 1.80 & & \\
\hline $\mathrm{G} 2 \times \mathrm{G} 4$ & -0.02 & -0.12 & 8.76 & 19.59 & 122.83 & 26.25 & -3.22 & -4.81 & & \\
\hline $\mathrm{G} 2 \times \mathrm{G} 7$ & -0.25 & $-1.78^{\star \star}$ & -16.69 & -14.00 & 112.69 & -111.97 & 2.32 & -1.40 & & \\
\hline $\mathrm{G} 2 \times \mathrm{G} 8$ & 0.76 & 0.16 & 4.34 & 25.23 & $189.94^{\star \star}$ & 13.16 & -1.18 & -5.78 & & \\
\hline G4 X G5 & 0.64 & 0.07 & 13.28 & 13.84 & $284.61^{\star \star \star}$ & -72.69 & -4.36 & $-7.92^{*}$ & & \\
\hline G4 X G8 & 0.37 & 0.52 & $41.90^{\star \star}$ & $30.34^{*}$ & 33.02 & $278.69^{*}$ & $15.39^{\star \star \star}$ & $7.71^{*}$ & & \\
\hline G6 X G8 & -1.20 & $-1.49^{\star *}$ & -5.47 & -1.53 & $175.22^{*}$ & $278.50^{*}$ & $19.30^{\star \star \star}$ & 4.29 & & \\
\hline
\end{tabular}

Table 6 (Continued) 


\begin{tabular}{|c|c|c|c|c|c|c|c|c|c|c|}
\hline \multirow[t]{2}{*}{ Cross } & \multicolumn{2}{|c|}{$\mathrm{PH}(\mathrm{cm})$} & \multicolumn{2}{|c|}{ DFW (days) } & \multicolumn{2}{|c|}{ DPM (days) } & \multicolumn{2}{|c|}{ DSF (days) } & \multicolumn{2}{|l|}{ NPPP } \\
\hline & DS & NS & DS & NS & DS & NS & DS & NS & DS & NS \\
\hline \multicolumn{11}{|c|}{ SCA effects of 5 bottom yielding crosses under drought stress } \\
\hline G1 X G4 & 0.08 & 4.52 & -1.62 & -1.67 & $-4.32^{*}$ & -2.78 & -3.81 & -1.29 & -2.68 & -4.65 \\
\hline G2 X G5 & -1.08 & $14.21^{*}$ & 1.50 & $3.29^{*}$ & 3.52 & 2.55 & 2.14 & -0.91 & -7.23 & -4.15 \\
\hline $\mathrm{G} 2 \times \mathrm{G} 6$ & 4.89 & 1.41 & -3.07 & -1.75 & -0.89 & $-2.80^{*}$ & 2.43 & -1.13 & -7.03 & -3.27 \\
\hline G4 X G7 & -13.92 & 1.56 & -1.66 & 2.76 & $4.42^{*}$ & 2.63 & $6.14^{*}$ & 0.03 & -3.58 & $-8.80^{*}$ \\
\hline G5 X G8 & -4.53 & $15.60^{* *}$ & 2.42 & $5.00^{\star \star}$ & $4.70^{\star}$ & $3.65^{\star \star}$ & 1.11 & -1.54 & -8.40 & $-13.06^{* \star}$ \\
\hline \multirow[t]{2}{*}{ Cross } & NSP & & NSPP & & GYD (g) & & & SW (g) & & \\
\hline & DS & NS & DS & NS & DS & NS & DS & NS & & \\
\hline \multicolumn{11}{|c|}{ SCA effects of 5 bottom yielding crosses under drought stress } \\
\hline G1 X G4 & 0.39 & 0.14 & 1.45 & -2.18 & -126.36 & -13.30 & $-5.80^{\star}$ & $-8.50^{*}$ & & \\
\hline G2 X G5 & -0.81 & -0.70 & -14.14 & -3.57 & -112.34 & -17.36 & 0.57 & 1.91 & & \\
\hline G2 X G6 & 0.84 & $2.08^{\star \star \star}$ & -20.28 & -3.41 & $-266.16^{\star \star \star}$ & -138.08 & $-8.30^{* *}$ & -3.96 & & \\
\hline G4 X G7 & -0.06 & 0.28 & -27.83 & -27.74 & -112.98 & -66.33 & 4.38 & -3.41 & & \\
\hline G5 X G8 & -0.76 & -0.49 & -18.18 & -31.42 & $-202.83^{\star \star}$ & $-296.91^{*}$ & $-12.12^{\star \star \star}$ & -4.42 & & \\
\hline
\end{tabular}

See footnote in Table 1 for genotype codes. DS moisture stressed environments, NS non-stressed environments, $P H$ plant height, $D F W$ days to flowering, DPM days to physiological maturity, DSF days to seed fill, NPPP number of pods per plant, NSP number of pods per pod, NSPP number of seeds per plant, GYD grain yield, $S W 100$ seed weight, * $p<0.05, * * p<0.01, * * * 0<0.001$

Two crosses, namely G4 X G8 and G6 X G8 showed significant $(p<0.05)$ and positive SCA effects for GYD, NSPP, and SW. Other good combiners with significant $(p<0.05)$ SCA effects for SW under NS conditions were G3 X G8 and G5 X G7. Good specific combiners for NSP were G1 X G5, G2 X $\mathrm{G} 3$ and $\mathrm{G} 2 \times \mathrm{G} 6$. For NPPP, significant $(p<0.05)$ and positive SCA effects were exhibited by the crosses $\mathrm{G} 2 \times \mathrm{G} 3, \mathrm{G} 4 \times \mathrm{G} 5$, and G4 $\times$ G8. Crosses $\mathrm{G} 2$ X G8, G3 X G5, G3 X G7, G5 X G6 and G5 X G8 showed significant $(p<0.05)$ and positive SCA for DPM. On the other hand, significant $(p<0.05)$ and negative SCA effects for DPM were exhibited by the crosses G1 X G7, G2 X G6, G5 X G7 and G6 X G8.

Association of grain yield with yield attributing traits under moisture stressed and optimal environments

Results of correlation coefficients among different traits under DS and NS conditions are presented in Table 7. Most of the correlations observed ranged from weak to strong. Significant and positive correlations were observed for NPPP $(r=0.19, p<0.05) \mathrm{PH}(r=0.21, p<0.05)$ and SW $(r=$ $0.36, p<0.001)$ with GYD under DS conditions. In addition, the NPPP was significantly and positively correlated with NSP ( $r=0.17, p<0.05$ ), NSPP $(r=0.60, p<0.001), \mathrm{PH}(r=0.40, p<0.001)$ and SW $(r=0.17, p<0.05)$ under DS conditions. Furthermore, significant and positive correlations were observed for NSP with NSPP $(r=0.26, p<0.01)$ under DS conditions. Under NS conditions, significant and positive correlations were observed for DPM $(r=0.96)$, NPPP $(r=0.33)$, NSPP $(r=0.42)$ and SW $(r=0.29)$ with grain yield. In addition, the NPPP was significantly $(p<0.001)$ and positively correlated with NSP $(r=0.32)$ and NSPP $(r=0.72)$ under NS conditions. Furthermore, significant $(p<0.001)$ and positive correlations were observed for NSP with NSPP $(r=0.34)$ under NS conditions.

Table 7 Pearson's correlation coefficients for pairwise comparison of nine traits among twenty-eight families and eight parents under moisture stress and non-moisture stress conditions in Zimbabwe 


\begin{tabular}{|c|c|c|c|c|c|c|c|c|c|}
\hline \multicolumn{10}{|c|}{ Under moisture stress conditions } \\
\hline Trait & DF & DPM & DSF & GYD & NPPP & NSP & NSPP & $\mathrm{PH}$ & SW \\
\hline DF & 1 & & & & & & & & \\
\hline DPM & $0.65^{\star \star \star}$ & 1 & & & & & & & \\
\hline DSF & $-0.33^{\star \star \star}$ & $0.51^{\star \star \star}$ & 1 & & & & & & \\
\hline GYD & $-0.19^{*}$ & $-0.16^{*}$ & 0.01 & 1 & & & & & \\
\hline NPPP & 0.05 & -0.05 & -0.11 & $0.19^{*}$ & 1 & & & & \\
\hline NSP & -0.13 & -0.16 & -0.05 & -0.01 & $0.17^{\star}$ & 1 & & & \\
\hline NSPP & 0.14 & 0.13 & 0.01 & 0.15 & $0.60^{\star \star \star}$ & $0.26^{* *}$ & 1 & & \\
\hline $\mathrm{PH}$ & 0.05 & -0.13 & $-0.21^{\star *}$ & $0.21^{*}$ & $0.40^{\star \star \star}$ & -0.05 & 0.11 & 1 & \\
\hline SW & $-0.37^{\star \star \star}$ & $-0.20^{\star \star}$ & $0.17^{*}$ & $0.36^{\star \star \star}$ & $0.17^{*}$ & -0.05 & 0.01 & 0.04 & 1 \\
\hline \multicolumn{10}{|c|}{ Under non-moisture stress conditions } \\
\hline Trait & DF & DPM & DSF & GYD & NPPP & NSP & NSPP & $\mathrm{PH}$ & SW \\
\hline DF & 1 & & & & & & & & \\
\hline DPM & $0.96^{\star \star \star}$ & 1 & & & & & & & \\
\hline DSF & -0.44 & -0.17 & 1 & & & & & & \\
\hline GYD & -0.26 & -0.19 & 0.29 & 1 & & & & & \\
\hline NPPP & -0.07 & -0.08 & -0.01 & $0.33^{\star \star \star}$ & 1 & & & & \\
\hline NSP & -0.13 & $-0.16^{*}$ & -0.05 & 0.10 & $0.32^{\star \star \star}$ & 1 & & & \\
\hline NSPP & -0.08 & -0.07 & 0.05 & $0.42^{\star \star \star}$ & $0.72^{\star \star \star}$ & $0.34^{\star \star \star}$ & 1 & & \\
\hline $\mathrm{PH}$ & $0.17^{*}$ & $0.18^{*}$ & -0.05 & 0.02 & -0.11 & -0.03 & -0.14 & 1 & \\
\hline SW & $-0.31^{\star \star \star}$ & $-0.27^{\star \star \star}$ & $0.23^{\star \star \star}$ & $0.29^{\star \star \star}$ & 0.14 & -0.03 & 0.06 & 0.01 & 1 \\
\hline
\end{tabular}

PH plant height, DFW days to flowering, DPM days to physiological maturity, DSF days to seed fill, NPPP number of pods per plant, NSP number of pods per pod, NSPP number of seeds per plant, GYD grain yield, SW100 seed weight, * $p<0.05,{ }^{\star \star} p<0.01$, ${ }^{\star \star \star} p<0.001$.

\section{Discussion}

The significant mean squares of genotypes observed in most of the traits under DS environments indicate the presence of wide genetic variability for drought tolerance among the parents and their progenies. This, therefore provides an opportunity to bean breeders to identify drought tolerant genotypes with desirable traits, which address the needs and preferences of farmers and processors. Several studies have previously reported such genetic variability for moisture stress tolerance among parents and progenies in dry beans (Makunde et al. 2007; Asadi et al. 2010; Habibi 2011; Amongi et al. 2015; Phiri 2015). Significant effects of water regimes on the performance of genotypes with respect to GYD, SW, and DSF suggested that moisture stress is associated with a reduction of the aforementioned traits. Thus, such interactions could cause changes in performance of genotypes between water regimes as was observed in the current study for certain genotypes.

The parents G3, G4, G6, G7, G8 and their progenies performed consistently well across the two water regimes with respect to NSP, NSPP, GYD, SW, NPPP, SW and DTI. This indicates that the aforementioned genotypes might have genetic factors conferring tolerance to moisture stress and are therefore likely to perform well in areas that are prone to drought stress. In self-pollinating crops such as navy bean, which have a lesser theoretical magnitude of heterosis, breeding programs should put more attention on additive gene effects that are fixable. Both additive and non-additive gene effects were important in the expression of traits under both environments as signified by the significant GCA and SCA effects for most of the studied traits. Traits such as DFW, DPM, DSF, and SW were influenced by additive gene action as shown by the existence of significant mean squares of GCA effects under both test environments. 
On the other hand, the significant mean squares of SCA observed under both test environments for traits such as DFW, DPM, NPPP, GYD, and SW indicate that non-additive gene action was also important in accounting for the expression of the aforementioned traits. This implies that artificial selection of these characters for further genetic improvement could be possible through artificial hybridization and recurrent selection methods (Owusu et al. 2017). The observed non-significant GCA and SCA effects for NSPP and NSP under NS environments suggest that both traits were influenced by epistatic gene effects. These findings are consistent with those by Goncalves et al. (2015) who observed non-significant SCA effects for NSP in an $\mathrm{F}_{2}$ dry bean population under NS conditions.

The significant interaction of genotypes, GCA and SCA with location for some of the traits indicates that different gene combinations, alleles and genes may be present for developing improved cultivars of navy bean that are adapted to moisture stress. For instance, "G1" exhibited positive and negative GCA effects for GYD under NS and DS environments respectively; thus, the gene/s influencing the expression of GYD under optimal conditions in "G1" did not contribute to moisture stress tolerance. Conversely, the parents "G3" and "G4" had positive (tolerant to moisture stress) and negative (poorly adapted to optimal conditions) GCA effects under DS and optimal environments. Similar findings were reported by Rainey and Griffiths (2005) in snap beans under high and low temperature environments and Chiipanthenga et al. (2021) in soybean (Glycine max L.) under DS and NS environments. Therefore, there is need to evaluate and select $F_{2}$ progenies independently (separately) across contrasting water regimes in order to identify superior and stable $F_{2}$ progenies that are tolerant to moisture stress. The GCA x Loc interaction effects were not significant for all the traits except for DFW (under DS) while SCA x Loc interaction effects were not significant for all the traits except for PH and NSPP (under DS) and PH and NSP (under NS) which was an indication of consistent expression of the studied traits across environments. These findings corroborate with the findings of Nkhata et al. (2021) who reported non-significant mean squares of GCA x Loc interaction effects for DFW, DPM, NSPP, NPPP, and GYD under optimal conditions in dry bean.

Several studies (Mwije et al. 2014; Nayak et al. 2018; Nkhata et al. 2021; Chiipanthenga et al. 2021) suggest the predominance of additive gene effects in the expression of a trait when Baker's ratio is more than 0.5. In the present study, Baker's ratio was greater than 0.5 for all characters under DS, NS and across all environments except for NPPP (under DS) and PH (across all environments), coupled with higher estimates of GCA variance (more than 1) over SCA for all the traits except for PH and NPPP under DS and NS environments, respectively. This suggests that both additive and non-additive gene action were important, with a preponderance of additive gene action in controlling most of the studied traits under DS, NS and across environments. Therefore, early generation selection for all the studied traits may be effective except for PH and NPPP. The present results agree with previous studies by Asadi et al. (2010) and Amongi et al. (2015) who observed the preponderance of additive gene action in the expression of GYD, pod weight, NSPP and NPPP in dry bean under moisture stressed conditions. Contrary to the current findings, lqbal et al. (2012) and Senbetay et al. (2015) observed the predominance of non-additive gene action for the expression of GYD, NSPP, and NPPP in dry bean under optimal environments. Differences in the genetic backgrounds of the parents used and the environments under which the experiments were conducted by the different researchers could be the reasons for the disparities in results with respect to the genetic control of certain traits. These conflicting results by several authors confirm that both additive and non-additive genes contribute to the expression of grain yield and its components in navy bean under DS and NS environments. This necessitates the need to harness both additive and non-additive types of genes through the population plant breeding approach (bi-parental mating) followed by reciprocal recurrent selection, backcross, pedigree method of selection and single seed descent in early filial generations of navy beans (Nayak et al. 2018).

Parental lines G3, G4, and G8 which consistently exhibited significant, positive, and high GCA effects for GYD and its attributing traits in a desirable direction under both environments are good general combiners. This suggests that they could possibly pass on the favourable alleles to their offspring during breeding which may result in transgressive segregants in subsequent segregating populations (Dholariya et al. 2014; Goncalves et al. 2015; Fasahat et al. 2016; Chiipanthenga et al. 2021). Furthermore, G3, G4, and G8 exhibited significant and negative GCA effects for DFW and DPM, indicating that they could also be useful in breeding for earliness in drought prone regions. Therefore G3, G4, and G8 could be useful in navy bean breeding programs to improve GYD and its components under both DS and NS environments. Parental lines such as G1, G7, and G8 showed higher GCA effects on GYD under optimal conditions than under DS conditions suggesting that the ability of genotypes to combine well depends on action, interaction, and linkage relationships of genes (Sofi et al. 2006). This highlights that superior GCA effects of genotypes under optimal conditions indicates their relative superiority under NS but does not necessarily indicate their ability to combine well with other genotypes under DS environments.

Under all the testing conditions, no single parent had high significant GCA effects for all the studied traits. Nevertheless, genetic recombination and gene pyramiding can be enhanced through several cycles of artificial hybridization and selection among the parental lines and their progenies to fix transgressive segregants (Joshi and Nayak 2010). Romanus et al. (2008) also suggested the use of various cross-combinations (double crosses, three-way crosses, and four-way crosses) other than single crosses in order to enhance genetic recombination. Mutation breeding through mutation induction and backcrossing breeding can also be used to enhance genetic recombination. The significant, positive, and high SCA effects for GYD observed under DS environments for cross-combinations G2 X G3, G2 X G8, G4 X G5, and G6 X G8 was an indication of transfer of favourable alleles for improved GYD performance from the parents to the progenies. Such crosses may result in transgressive segregants that could be selected for DS environments. Thus, these crosses represent potential breeding material to further select for improved GYD and yield attributing traits under DS and NS environments. The superior performance of these crosses may be attributed to the involvement of additive $x$ dominance (high/low - G2 X G8; G4 X G5; G6 X G8), and additive x additive (high/high - G4 X G8) type of gene action interactions for expression of GYD and its 
components. In studies by lqbal et al. (2012) on dry bean, most of the superior cross-combinations under optimal environments involved high GCA x low GCA, average GCA x low GCA, high GCA x average GCA and average GCA x average GCA general combiners.

Over dominance, wide genetic base and complimentary epistatic gene effects (non-allelic interaction) at heterozygous loci could be attributed to the superiority of progenies from parents with low GCA effects (Girase and Deshmukh 2000; Nayak et al. 2018). Similar findings were observed by Goncalves-Vidigal et al. (2008) and Senbetay et al. (2015) who reported that parental genotypes with low GCA effects resulted in superior hybrids in dry bean under optimal conditions. These findings imply that parental genotypes should not be discarded from the breeding pipeline based on only unfavourable and negative GCA estimates. Interestingly, some of the parents with high and positive GCA effects for some of the traits produced progenies with unfavourable negative SCA effects under both DS and NS environments. Similar findings were reported by Mwije et al. (2014) and Musembi et al. (2015) in sweet potato (Ipomea batatas L. Lam.) and Chiipanthenga et al. (2021) in soybean. These findings could be attributed to the lack of genetic complementation between genes of the parents involved and the presence of modifier genes which may act in combination resulting in large phenotypic variability. This suggests that high GCA effects of the parental lines indicate their relative superiority but does not necessarily mean that crossing of parental lines with high GCA effects will result in progenies with significant, positive, and high SCA effects.

The significant positive correlations that were observed between GYD and yield components (NPPP, NSPP, and SW) under both environments suggest that these traits can be improved simultaneously. Therefore, GYD improvement in navy bean under both DS and NS environments can be achieved through both direct and indirect selection for NPPP, NSPP and SW. Similar findings were reported by Romanus et al. (2008) in cowpeas with respect to DF, DSF, and DPM under optimal conditions.

\section{Conclusions, Recommendations, And Implications For Breeding}

There is potential for breeding progenies with superior drought tolerance, which can improve GYD performance under DS environments in Zimbabwe. Both additive and non-additive gene effects were important in the inheritance of GYD and its attributing traits with preponderance of additive gene action under both test environments. This implies that there is need to incorporate breeding schemes that exploit both additive and non-additive genes in navy bean breeding. Parents G3, G4, G7, and G8 were the best combiners for GYD and its components under DS. These parents could be utilized in navy bean improvement programs to form base populations with improved tolerance to drought. The best performing specific combiners with consistent high values for most of the studied traits under both environments were G2 X G3, G2 X G8, G4 X G5, G4 X G8, and G6 X G8. Among these, G4 X G8 and G6 X G8 had high DTI under both DS and NS environments. Thus, potential lines with improved tolerance to drought can be selected from these promising crosses for further evaluation before release. The significant and positive correlations of GYD with NPPP, NSPP, and SW suggest the feasibility of indirect selection for GYD through secondary traits. Grain yield was significantly and positively correlated with NPPP, NSPP, and SW under both optimal and moisture stressed environments suggesting that these traits can be improved simultaneously. Grain yield, NPPP, NSPP, and SW were identified as best selection criteria for utilization in navy bean breeding. Breeding for superior grain yield under DS should involve high GCA x high GCA or high GCA x low GCA parental combinations. Parental genotypes should not be discarded from the breeding pipeline based on only unfavourable and negative GCA estimates. High GCA effects of the parental lines indicate their relative superiority but do not necessarily mean that crossing of parental lines with high GCA effects will result in progenies with significant, positive, and high SCA effects.

\section{Declarations}

Acknowledgements The authors thank the Swiss Agency for Development and Cooperation (SDC) and the Pan Africa Bean Research Alliance (PABRA) for financial and technical support of the study respectively. We also thank the Alliance of Bioversity International and International Centre of Tropical Agriculture (CIAT) for providing germplasm. Mention also goes to Crop Breeding Institute, Chiredzi Research Station, and Chisumbanje Experiment Station for providing research space.

\section{Compliance with ethical standards}

Conflict of interest Authors have no conflict of interest to declare.

Author contributions BM was involved in the study conception, establishment of the research work, phenotyping, data analysis, and drafting of the manuscript. JS (major supervisor) and EG (co-supervisor) were involved in the designing and supervision of the experiments, data interpretation and critically revising the manuscript. KS, AK, and PM were involved in critically reviewing the manuscript. All authors' read and approved final manuscript.

\section{References}

1. Acquaah G (2009) Principles of Plant Genetics and Breeding. John Wiley \& Sons, USA

2. AGRITEX (2016) Second round crop and livestock assessment report. http://www.cfuzim.com/wpcontent/uploads/2017/06/june17production.pdf. Accessed on 25 May 2018

Page $15 / 17$ 
3. Allard RW (1960) Principles of Plant Breeding. John Wiley and Sons. Inc., New York, United States

4. Amongi W, Nkalubo ST, Gibson P, Edema R, Ochwo-Ssemakula M (2015) Genetics of drought tolerance in common bean genotypes adapted to Ugandan conditions. J Plant Breed Crop Sci 7:18-27. https://doi.org/10.5897/JPBCS2014.0462

5. Aruda IM, Modacirino V, Koltun A, Zeffa DM, Nagashima GT, Goncalves LSA (2019) Combining agro-morphological and physiological traits in different gene pools of common bean subjected to water deficit. Agronomy 9:371-383. https://doi.org/10.3390/agronomy9070371

6. Asadi B, Bihamta MR, Dori HR (2010) Genetic analysis of drought tolerance in white bean. Seed Plant Improv J 26:469-484

7. Assefa T, Beebe S, Rao IM, Cuasquer J, Duque MC, Rivera M, Battisti A, Lucchin M (2013) Pod harvest index as a selection criterion to improve drought resistance in white pea bean. Field Crops Res 148:24-33. https://doi.org/10.1016/j.fcr.2013.04.008

8. Baker RJ (1978) Issues in diallel analysis. Crop Sci 18:533-536. https://doi.org/10.2135/cropsci1978.0011183X001800040001x

9. Bartlett MS (1947) The use of transformations. Biometrics 3:39-52. https://doi.org/10.2307/3001536

10. Beebe SE, Rao IM, Blair M, Acosta-Gallegos JA (2010) Phenotyping common beans for adaptation to drought. In: Ribaut JM, Monneveux P (Eds.) Drought Phenotyping in Crops: From Theory to Practice. Generation Challenge Program Special Issue on Phenotyping. pp. $311-334$

11. Beebe SE, Rao IM, Blair MW, Acosta-Gallegos JA (2013) Phenotyping common beans for adaptation to drought. Front Physiol 4:1-20. https://doi.org/10.3389/fphys.2013.00035

12. Chiipanthenga MK, Labuschagne MT, Fandika IR, van der Merwe R (2021) Combining ability of soybean (Glycine max L.) yield performance and related traits under water-limited stress conditions. Euphytica 217:41. https://doi.org/10.1007/s10681-021-02780-5

13. Dabholkar AR (1992) Elements of biometrical genetics. Concept publishing company, New Delhi, India

14. Darkwa K, Ambachewa D, Mohammed H, Asfawa A, Blair MW (2016) Evaluation of common bean (Phaseolus vulgaris L.) genotypes for drought stress adaptation in Ethiopia. Crop J 4:367-376. https://doi.org/10.1016/j.cj.2016.06.007

15. Dholariya N, Akabari V, Patel J, Chovatia V (2014) Combining ability and gene action study for grain yield and its attributing traits in bread wheat. Electron J Plant Breed 5:402-407

16. Fasahat P, Rajabi A, Rad JM, Derera J (2016) Principles and utilization of combining ability in plant breeding. Biom biostat int j 4:1-24. https://doi.org/10.15406/bbij.2016.04.00085

17. Girase VS, Deshmuck RB (2000) Gene action for yield and its components in chickpea. Indian J Genet Plant Breed 60:185-189

18. Goncalves JGR, Chiorato AF, da Silva DA, Esteves JAE, Bosetti F, Carbonell SAM (2015) Combining ability in common bean cultivars under drought stress. Bragantia 74:149-155. http://dx.doi.org/10.1590/1678-4499.0345

19. Goncalves-Vidigal MC, Silverio L, Elias HT, Filho PSV, Kvitschal MV, Retuci VS, da Silva CR (2008) Combining ability and heterosis in common bean cultivars. Pesqui Agropecu Bras 43:1143-1150

20. Griffings B (1956) Concept of general and specific combining ability in relation to diallel crossing systems. Aust J Biol Sci 9:463-493. https://doi.org/10.1071/BI9560463

21. Habibi G (2011) Influence of Drought on Yield and Yield Components in White Bean. International Journal of Agricultural and Biosystems Engineering 5:380-389. https://doi.org/10.5281/zenodo.1079518

22. Iqbal AM, Nehvi FA, Wani SA, Dar ZA, Lone AA, Qadri H (2012) Combining ability study over environments in dry beans (Phaseolus vulgaris L.). SAARC J Agric 10:61-69. https://doi.org/10.3329/sja.v10i2.18324

23. Joshi R, Nayak S (2010) Gene pyramiding-A broad spectrum technique for developing durable stress resistance in crops. Biotechnol Mol Biol Rev 5:51-60

24. Katungi E, Mutua M, Mutari B, Makotore, Kalemera WS, Maereka E, Zulu R, Birachi E, Chirwa R (2017) Improving bean production and consumption in Zimbabwe baseline report. https://cgspace.cgiar.org/bitstream/handle/10568/82724/Baseline_report_for_Zimbabwe_final.pdf?sequence=1. Accessed on 30 April 2018

25. Makunde GS (2007) Common bean breeding procedures. In: Nyakanda P, Hlungwani M, Manzira C, Ngirazi S, Mushoriwa H (eds) Practical crop improvement methodologies in Zimbabwe. Prinflow, Harare, pp 51-53

26. Makunde GS (2013) Quantification of genetic diversity for drought adaptation in a reference collection of common bean (Phaseolus vulgaris L.). PhD Thesis, University of Free State. Bloemfontein

27. Makunde GS, Beebe S, Blaire MW, Chirwa R, Lungu D (2007) Inheritance of drought tolerance traits in Andean x Andean and Andean $x$ Mesoamerican F2 populations. Annu rep Bean Improv Coop 50:159-160

28. Mulugeta A, Hussein M, Habtamu Z (2013) Inheritance of primary yield component traits of common beans (Phaseolus Vulgaris L.): Number of seeds per pod and 1000 seed weight in an $8 \times 8$ diallel cross population. Int J Genet Mol Bio 5:42-48

29. Mutari B, Sibiya J, Bogweh Nchanji E, Simango K, Gasura E (2021) Farmers' perceptions of navy bean (Phaseolus vulgaris L.) production constraints, preferred traits and farming systems and their implications on bean breeding: a case study from South East Lowveld region of Zimbabwe. J Ethnobiol Ethnomed 17:13. https://doi.org/10.1186/s13002-021-00442-3

30. Musembi KB, Githiri SM, Yencho GC, Sibiya J (2015) Combining ability and heterosis for yield and drought tolerance traits under managed drought stress in sweet potato. Euphytica 201:423-440. http://doi.org/10.1007/s10681-014-1230-1

Page $16 / 17$ 
31. Mwinje A, Mukasa SB, Gibson P, Kyamanywa S (2014) Heritability analysis of putative drought adaptation traits in sweet potato. Afr Crop Sci J 22:79-87

32. Nayak NJ, Maurya PK, Maji A, Mandal AR, Chattopadhyay A (2018) Combining ability and genetic control of pod yield and component traits in dolichos bean. Int J Veg Sci 24:390-403. https://doi.org/10.1080/19315260.2018.1423598

33. Nkhata W, Shimelis H, Melis R, Chirwa R, Mzengeza T, Mathew I, Shayanowako A (2021) Combining ability analysis of common bean (Phaseolus vulgaris L.) genotypes for resistance to bean fly (Ophiomyia spp.), and grain yield and component traits. Euphytica 217:93. https://doi.org/10.1007/s10681-021-02833-9

34. Owusu GA, Nyadanu D, Obeng-Antwi K, Amoah RA, Danso FC, Amissah S (2017) Estimating gene action, combining ability and heterosis for grain yield and agronomic traits in extra-early maturing yellow maize single-crosses under three agro-ecologies of Ghana. Euphytica $213: 287$. https://doi.org/10.1007/s10681-017-2081-3

35. Payne RW, Murray DA, Harding SA (2018) An introduction to GenStat command language. VSN International, Hampstead, UK

36. Phiri $\mathrm{P}$ (2015) Genetic analysis of common bean (Phaseolus vulgaris L.) genotypes for tolerance to drought and heat stress in Zambia. PhD Thesis, University of KwaZulu Natal. South Africa

37. Porch TG, Bernsten R, Rosas JC, Molly J (2007) Climate change and the potential economic benefits of heat tolerant bean varieties for farmers in Atlantida, Honduras. J Agric Univ P R 91:133-148. https://doi.org/10.46429/jaupr.v91i3-4.3281

38. Rainey K, Griffiths P (2003) Evaluation of common bean yield components under heat stress. HortScience 38:682

39. Rainey KM, Griffiths PD (2005) Differential response of common bean genotypes to high temperature. J Am Soc Hortic Sci 130:18-25. https://doi.org/10.21273/JASHS.130.1.18

40. Robinson HF, Comstock RE, Harvey PH (1949) Estimation of heritability and the degree of dominance in corn. Agron J 41:353-359. https://doi.org/10.2134/agronj1949.00021962004100080005x

41. Rodriguez F, Alvarado G, Pacheso A, Burgueno A, Crossa J (2015) Analysis of genetic designs with R for Windows, version 3.0. CIMMYT Research Data and Software Repository

42. Romanus KG, Hussein S, Mashela WP (2008) Combining ability analysis and association of yield and yield components among selected cowpea lines. Euphytica 162:205-210. https://doi.org/10.1007/s10681-007-9512-5

43. Senbetay T, Tesfaye A (2015) Combining ability of commercial white pea bean (Phaseoulus vulgaris L.) varieties in South Western Ethiopia. J boil agric healthc 5:153-161

44. Singh SP (2001) Broadening the genetic base of common bean cultivars: A review. Crop Sci 41:1659-1675. https://doi.org/10.2135/cropsci2001.1659

45. Sofi P, Rather AG, Wani SA (2006) Combining ability and gene action studies over environments in field pea (Pisum sativum L.). Pak J Biol Sci 9:2689-2692. https://doi.org/10.3923/pjbs.2006.2689.2692

46. Sprague GF, Tatum LA (1942) General versus specific combining ability in single crosses of corn. J Am Soc Agron 34:923-932. https://doi.org/10.2134/agronj1942.00021962003400100008x

47. Su J, Zhang F, Yang X, Wu Y, Guang Z, Fan W (2017) Combining ability, heterosis, genetic distance and inter-correlations for waterlogging tolerance traits in chrysanthemum. Euphytica 213:1-5. https://doi.org/10.1007/s10681-017-1837-0

48. Teich H (1984) Heritability of grain yield, plant height and test weight of a population of winter wheat adapted to Southwestern Ontario. Theor Appl Genet 68:21-23. https://doi.org/10.1007/BF00252304

49. The Integrated Breeding Platform's Breeding Management System (2021) The Integrated Breeding Platform version 18. https://www.integratedbreeding.net/breeding-management-system. Accessed on 10 June 2021

50. Tsiko S (2018) New bean variety could save Zim US\$120K a month. http:// www.herald.co.zw/new-bean-variety-could-save-zim-us120k-amonth/amp/ . Accessed on 24 May 2020

\section{Supplementary Files}

This is a list of supplementary files associated with this preprint. Click to download.

- OnlineResource1.docx

- OnlineResource2.docx

- OnlineResource3.docx 